\title{
Confortement des ouvrages en terre par la technique du clouage vertical sur le réseau ferré national
}

\section{TALFUMIÈRE}

SNCF

6. avenue

François-Mitterrand

93574 La Plaine Saint-Denis vincent.talfumiere@sncf.fr
Les ouvrages en terre sur le réseau ferré français sont le siège de désordres dont l'évolution peut à terme menacer la sécurité et la régularité des circulations ferroviaires. Depuis plus de 30 ans, la SNCF a développé avec le LCPC des techniques de confortement par clouage vertical battu ou foré (clous unitaires) qui permettent de s'affranchir de l'achat de terrains et de stabiliser des surfaces de rupture profondes pouvant concerner le sol support. Le confortement est dimensionné à partir de la méthode des modules de réaction; le pieu va se déformer et il se crée un effort résistant au niveau de la surface de rupture qui va s'opposer au mouvement des terrains pris dans le glissement. Il faut attendre que le sol se déforme pour que le confortement soit actif donc des déplacements résiduels après travaux sont observés. Cet article précise la méthode de dimensionnement à partir d'essais pressiométriques, dans le contexte d'une infrastructure ferroviaire (dimensionnement intégrant les déformations admissibles de la voie, les sujétions de réalisation, etc.). Un exemple de confortement est présenté comprenant les différentes phases d'étude, les travaux avec leurs contraintes de mise en œuvre, et les contrôles nécessaires.

Mots-clés : infrastructure ferroviaire, confortement par clouage, essai pressiométrique, méthode de dimensionnement, contrôle.

\section{Reinforcement of earthworks by vertical nailing technique in the national railway network}

The earthworks on the French railway network undergo disorders whose evolution can in the long term threaten the safety and regularity of railway traffics. Since more than 30 years, the SNCF has developed with the LCPC the techniques of reinforcement by beaten or drilled vertical nails (unit nails), that make it possible to avoid land acquisition and to stabilize deep failure surfaces that could involve sub-soils. The reinforcement is designed using the method based on the modulus of reactions; the pile will deform so that it creates an effort resistant to the failure surface and opposed to the sliding movement. It should be waited until the ground deforms so that the reinforcement becomes active; thus residual displacements after earthwork are observed. This paper specifies the design method based on the pressiometer tests, in the context of a railway infrastructure (dimensioning integrating the admissible deformations of the railway, operation subjection, etc.) an example of reinforcement is presented, including the various phases study; the earthwork with their operation constraints; and the needed controls. 


\section{Introduction}

Les ouvrages en terre sont susceptibles de voir leur condition de stabilité s'altérer avec le temps, à cause de facteurs internes ou externes, qui peuvent entraîner des déformations irréversibles avec détérioration de la géométrie de la voie ferrée. Lorsque le désordre est détecté, un suivi des mouvements affectant l'ouvrage en terre est entrepris par la SNCF, à qui RFF, propriétaire du réseau, en délègue la maintenance en tant que GID (gérant de l'infrastructure délégué). En fonction de l'évolution du site, des travaux sont définis et réalisés afin de traiter l'ouvrage préventivement avant une éventuelle accélération qui aurait des conséquences sur la régularité ou sur la sécurité des circulations ferroviaires.

Le patrimoine ouvrages en terre du réseau ferré national est constitué d'une multitude de types d'ouvrages (remblais, déblais meubles ou rocheux, ouvrages en profil mixte...) intégrés dans un environnement particulier et évolutif (urbain, contexte hydraulique, versant rocheux, infrastructures accolées, zones de cavités...). C'est la raison pour laquelle lorsque cet ouvrage subit des mouvements et doit faire l'objet d'un traitement, une multitude de critères et de contraintes président au choix de la solution confortative.

Ce choix doit aussi tenir compte de la particularité du domaine ferroviaire par rapport aux autres infrastructures de transport :

- les contraintes propres aux circulations ferroviaires (nécessité de maintenir le trafic) ;

- les difficultés d'accès (choix de techniques et de matériels adaptés);

- la nécessité de garantir la sécurité des biens et des personnes transportés (contrat entre la SNCF et le voyageur) ;

- l'utilisation de méthodes de confortement et de prescriptions d'exécution adaptées au contexte spécifique de l'exploitation ferroviaire.

Les ouvrages en terre qui sont le siège de désordres sont recensés, connus et suivis. Ils font l'objet d'un classement (ouvrages en terre sensibles), qui suivant le référentiel interne à la SNCF, entraîne la rédaction d'une fiche signalétique descriptive de l'ouvrage en terre et la réalisation d'une visite détaillée au maximum quinquennale permettant d'observer l'évolution du site et de faire le point sur les suivis (inclinométriques, piézométriques, topographiques, etc.) de l'ouvrage à un instant $t$. Une surveillance, des travaux d'entretien ou de régénération sont préconisés suite à cette visite ou suite à une expertise nationale.

Parmi l'ensemble des techniques de confortement, il peut être recommandé de traiter l'ouvrage par clouage vertical battu ou foré qui est une solution couramment utilisée dans le domaine ferroviaire, et dont les premiers développements datent du début des années 1980 en collaboration avec le LCPC (Cartier, 1986 ; Frank, 1994). Le premier site expérimental a été étudié en 1982 au niveau du remblai de Boussy-SaintAntoine où le confortement par pieux de gros diamètres $(800 \mathrm{~mm})$ a été suivi en phase de réalisation et dans la phase de stabilisation durant les années qui ont suivi. Il a été démontré une diminution très importante des mouvements affectant l'ouvrage dans les deux années après travaux de confortement. La méthode du clouage vertical a vu une utilisation croissante dans le domaine ferroviaire de par la configuration des sites à conforter :

- accès souvent difficiles ;

- absence d'emprises en pied, parfois contexte urbain ;

- bonne adaptation du procédé pour des glissements de versant, ou dans un contexte de glissement sur sols compressibles.

Dans la suite de l'article, cette méthode est décrite plus précisément, en prenant en exemple le traitement d’un remblai sur versant.

\section{2}

\section{Exemple du confortement du remblai des Bas}

\section{9.}

\section{Présentation}

La ligne ferroviaire de Mantes-la-Jolie à Cherbourg traverse la Haute et la Basse-Normandie sur environ $300 \mathrm{~km}$ et constitue la desserte Paris-Cherbourg. C'est une ligne à double voie qui a été électrifiée en 1996 , avec augmentation des vitesses jusqu'à $200 \mathrm{~km} / \mathrm{h}$ sur certains tronçons. Pour améliorer la fluidité du trafic dans le cadre de cette modernisation, des installations permanentes de contresens ont été mises en place, constituées d'un appareil d'aiguillage pour passer d'une voie sur l'autre tous les 20 à $30 \mathrm{~km}$ et d'une signalisation adaptée.

Au niveau du hameau des Bas, au km 203, à $13 \mathrm{~km}$ à l'ouest de Lisieux (plan de situation en figure 1), la plate-forme ferroviaire est établie en remblai sur versant, d'une hauteur de 2 à $3 \mathrm{~m}$ côté gauche (amont) et de $7 \mathrm{~m}$ de hauteur côté droit (aval). La vitesse maximum sur ce secteur est de $150 \mathrm{~km} / \mathrm{h}$. Un appareil de voie se situe juste dans la zone concernée pour passer d'une voie sur l'autre. Le contexte géologique de cet ouvrage en terre est très défavorable; il repose en effet sur des marnes de l'Oxfordien de très mauvaise qualité qui ont entraîné de nombreux désordres sur le tronçon

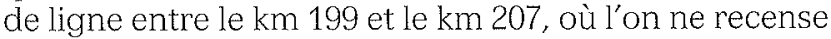
pas moins de 15 parties d'ouvrages sensibles.

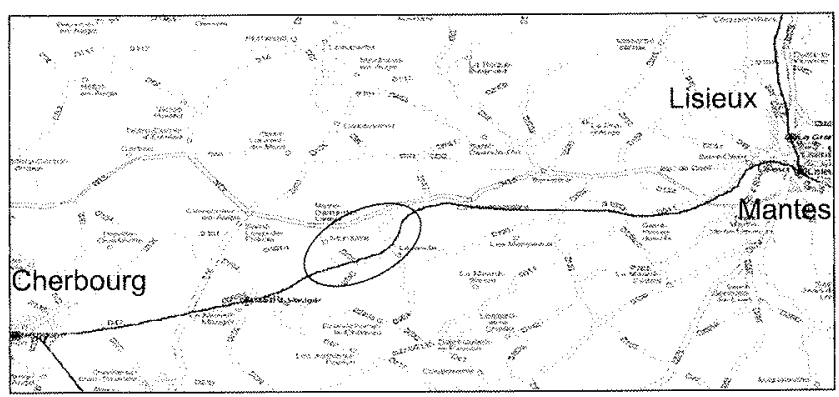

HC. 1 Plan de situation de la zone instable. Location of the instable zone. 


\section{Détection et expertise des désordres}

Les désordres affectant cet ouvrage ont été détectés en 2000, suite au passage de la voiture Mauzin (qui enregistre la géométrie de la voie). Un suivi du nivellement des deux voies a été entrepris par l'établissement Infrastructure de Caen, qui a montré le tassement d'une des deux voies (la voie côté droit aval) sur une soixantaine de mètres.

Une expertise sur site a été menée par le service Ouvrages en terre de la direction de l'Ingénierie pour diagnostiquer les désordres. Cette expertise consistait à :

- examiner l'ensemble des indices de désordres dans les emprises ferroviaires, mais également, à proximité des emprises, dans l'environnement ; cette visite se fait après un débroussaillage partiel ou total de l'ouvrage ou en période hivernale, afin d'optimiser les observations ;

- corréler les évolutions des suivis avec les observations faites lors de l'expertise;

- préconiser des suivis complémentaires et/ou une instrumentation avec reconnaissance des sols préalables pour expliquer les phénomènes affectant l'ouvrage en terre;

- définir des mesures de surveillance de l'ouvrage ffréquence de visite intermédiaire, fréquence des suivis, conditions de surveillance en période d'intempéries) et les travaux éventuels d'entretien ou de régénération à engager.
L'expertise a permis d'observer la présence de (voir Figs. 2 à 5) :

- un affaissement prononcé de l'accotement de la plateforme (appelé ( piste ) dans le vocabulaire ferroviaire) au droit des désordres mesurés en voie ;

- un déversement des supports caténaires (supports métalliques dont la fondation en béton a une profondeur de l'ordre de 2 mètres sous la piste et situés tous les 50 à $60 \mathrm{~m}$, portant le fil caténaire où un courant alternatif $25000 \mathrm{~V}$ circule) situés dans la zone de tassement;

- des déformations (gonflements et bourrelets) dans le champ à l'aval de l'ouvrage en terre, le tout dans un contexte de versant moutonné ;

- un drainage amont étanche (béton) en bon état ;

- un drainage aval mal marqué ;

- cles confortements existants de part et d'autre de l'ouvrage instable, datant de plusieurs dizaines d'années, montrant l'activité du site par le passé.

\section{9.}

\section{Suivis de l'ouvrage}

\section{5e.}

\section{Suivi topographique}

Lorsqu'un Ouvrage en terre évolue, il est nécessaire de suivre le nivellement des voies affectées par ce mouvement et de les remettre à niveau par introduction de

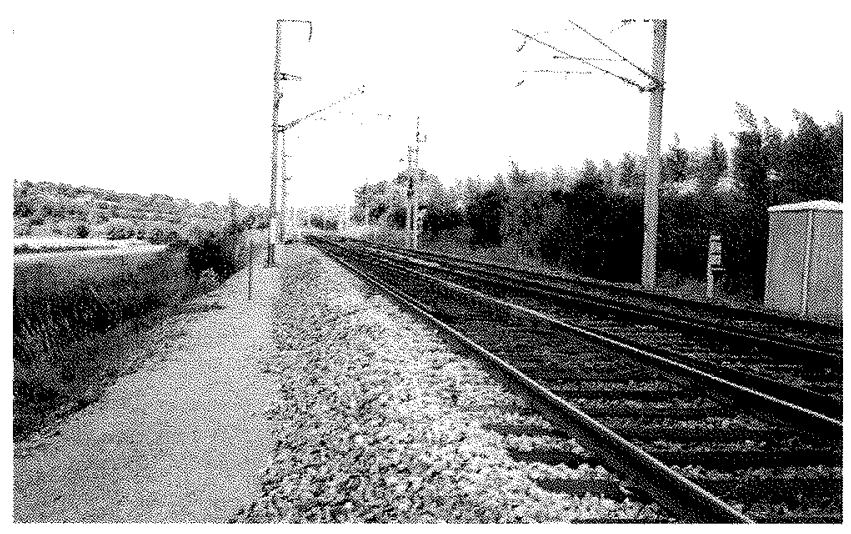

1. d'aiguillage.

Overall view of the site at referral device.

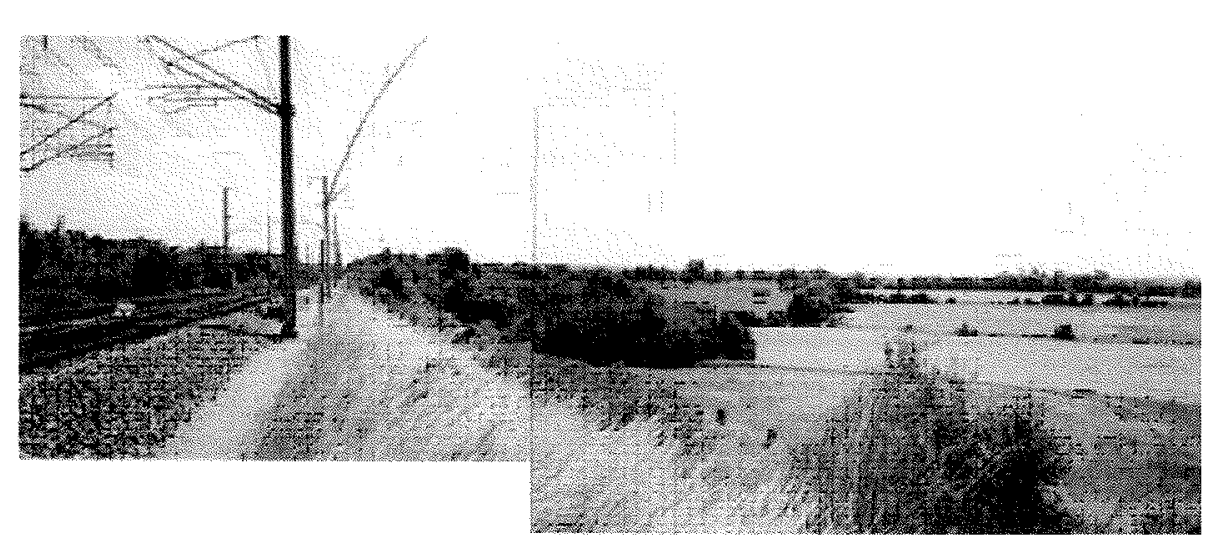

HG. 3 Vue générale des désordres.

Overall view of damages. 


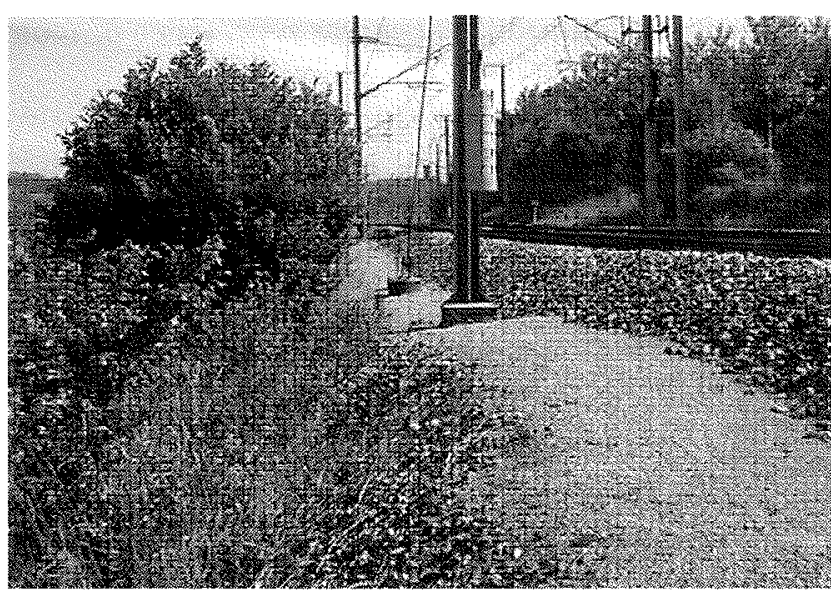

16.4 Vue de l'affaissement prononcé de la piste. View of significant settlement of track.

ballast sous les traverses, intervention mécanique que l'on appelle bourrage. Pour suivre au mieux ces mouvements, il est nécessaire de faire un levé topographique de l'altitude des files de rail avant l'intervention de bourrage puis d'en faire un deuxième après cette intervention; la différence sera représentée sur un graphique qui comptabilisera par une cumulée l'ensemble des informations sur un même secteur. Ce graphique permet d'obtenir l'extension de la zone de désordres et son activité en fonction du temps (Fig. 6). Les mouvements montrent une activité importante avec un tassement de $7 \mathrm{~mm}$ par mois, sur les deux files de rail de la voie droite (côté aval).

L'instrumentation de l'ouvrage en terre en inclinométrie et en piézométrie sur le ou les profils les plus défavorables (ceux qui sont le siège des mouvements les plus importants, Fig. 7) est également réalisée.

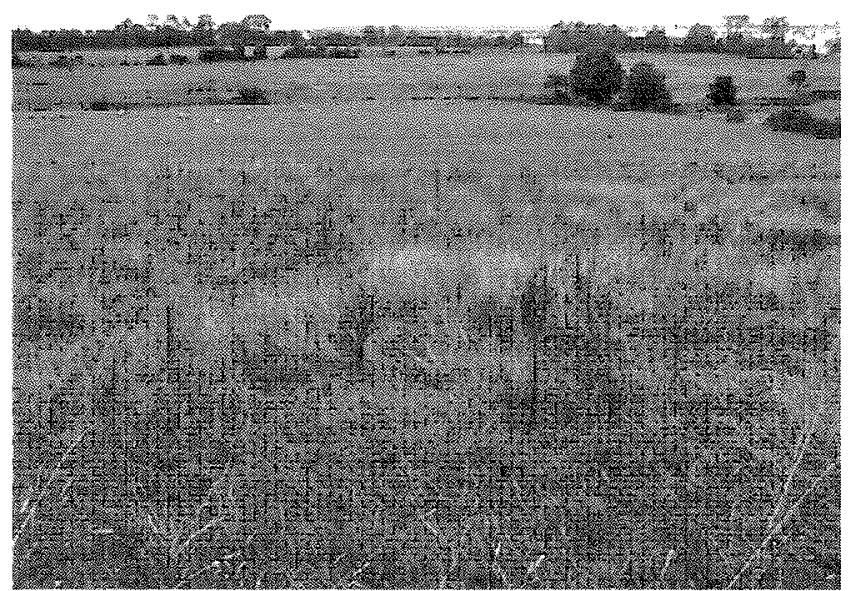

Ha. 5 Vue des bourrelets à l'aval du remblai dans le versant.

View of beads in downstream of the embankment in the slope.

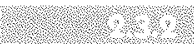 \\ Suivi inclinométrique}

Les tubes inclinométriques posés en 2002 ont été suivis tous les quatre mois; le résultat de ces mesures montre:

- sur le tube I1 côté gauche de la plate-forme en piste: pas de mouvement (Fig. 8);

- sur le tube I2 côté droit en piste : une rupture active à $5 \mathrm{~m}$ de profondeur (Fig. 9) ;

- sur le tube 13 en pied de talus côté droit : une rupture active à $3 \mathrm{~m}$ de profondeur (Fig. 10) ;

- sur le tube I4 à $20 \mathrm{~m}$ à l'aval du pied de talus dans le champ riverain : une rupture active à $3,50 \mathrm{~m}$ de profondeur (Fig. 11).
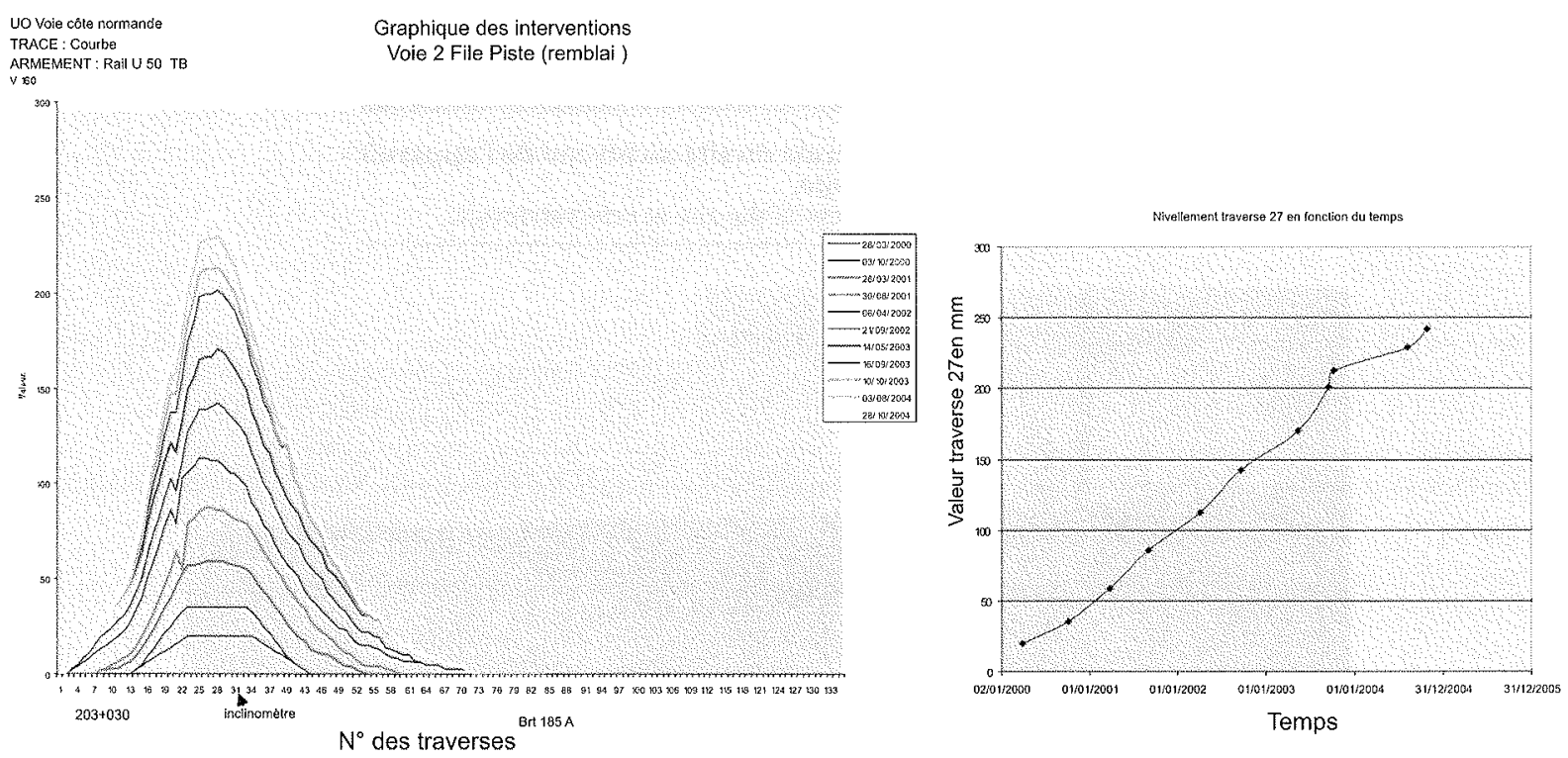

FI. 8 Graphique de cumulées de reprises de nivellement sur le site instable et évolution en fonction du temps.

Plot of cumulative level retrieving at the instable site and its evaluation versus time. 


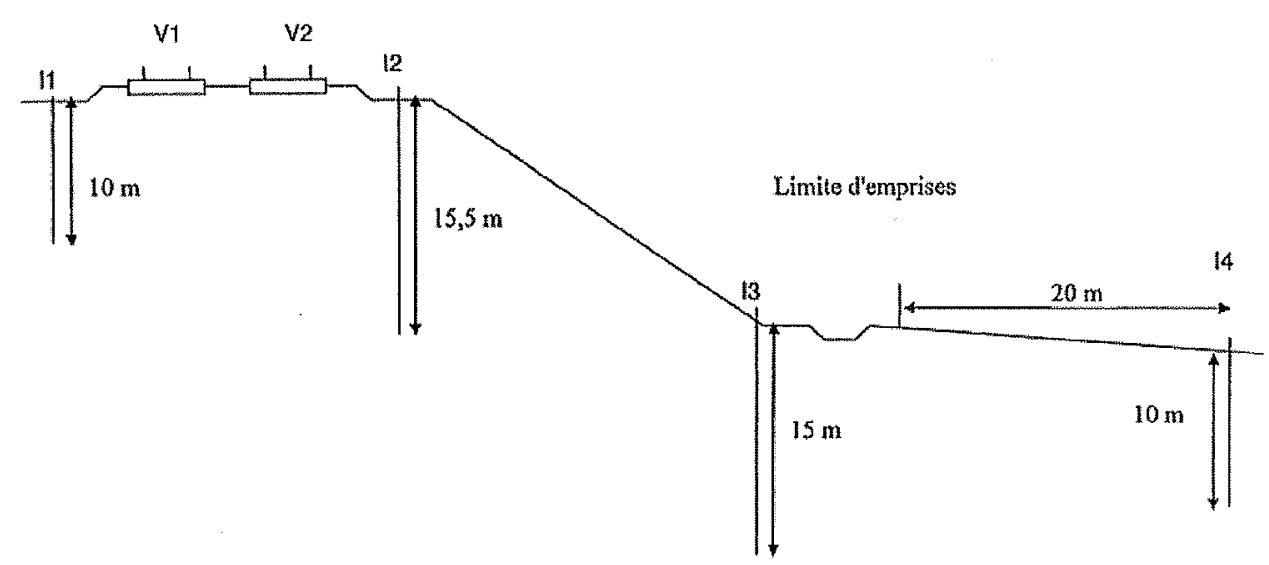

fic. 7 Plan d'implantation de l'instrumentation inclinométrique. Locations of inclinometers.

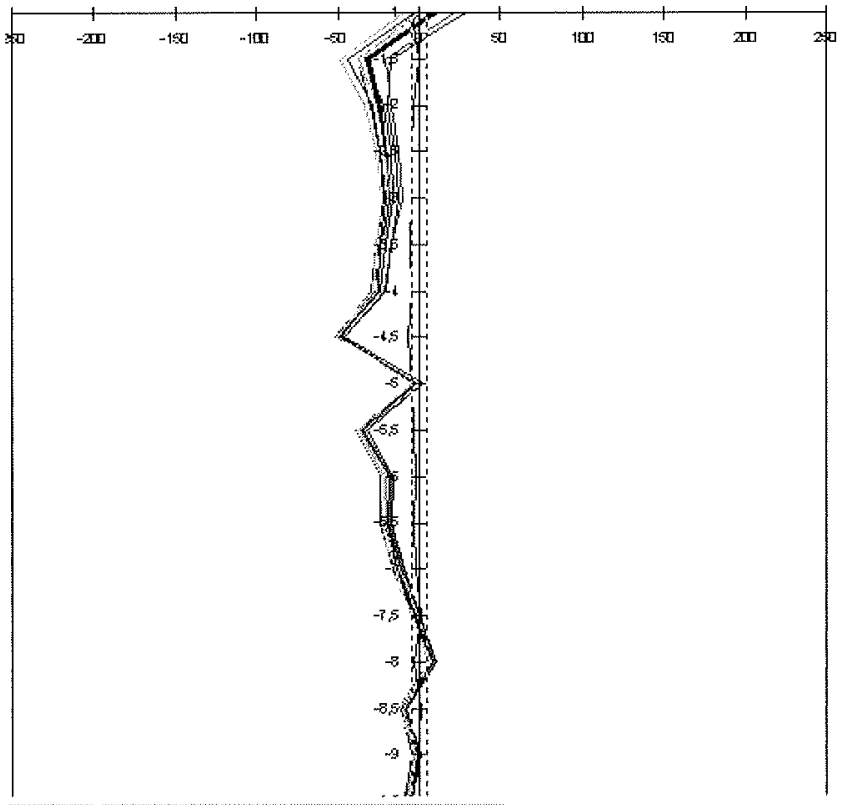

116. 8

Dépouillement angulaire du tube inclinométrique I1 (côté gauche amont).

Angular interpretation of tube inclinometer I1 (left side upstream).

Le déplacement latéral moyen des 3 tubes, côté droit, est de $5 \mathrm{~mm}$ par mois, ce qui est important pour ce type d'ouvrage et compte tenu de la période de mesure ( 1 an et demi).

\section{4}

\section{Décision de traitement}

Plusieurs critères nous ont amenés à envisager un traitement rapide de l'ouvrage en terre sur l'ensemble de la zone montrant des indices de désordres, à savoir une centaine de mètres linéaires de plate-forme:

- forte activité des mouvements affectant le site $(7 \mathrm{~mm} /$ mois en topographie et $5 \mathrm{~mm} /$ mois en inclinométrie) ;

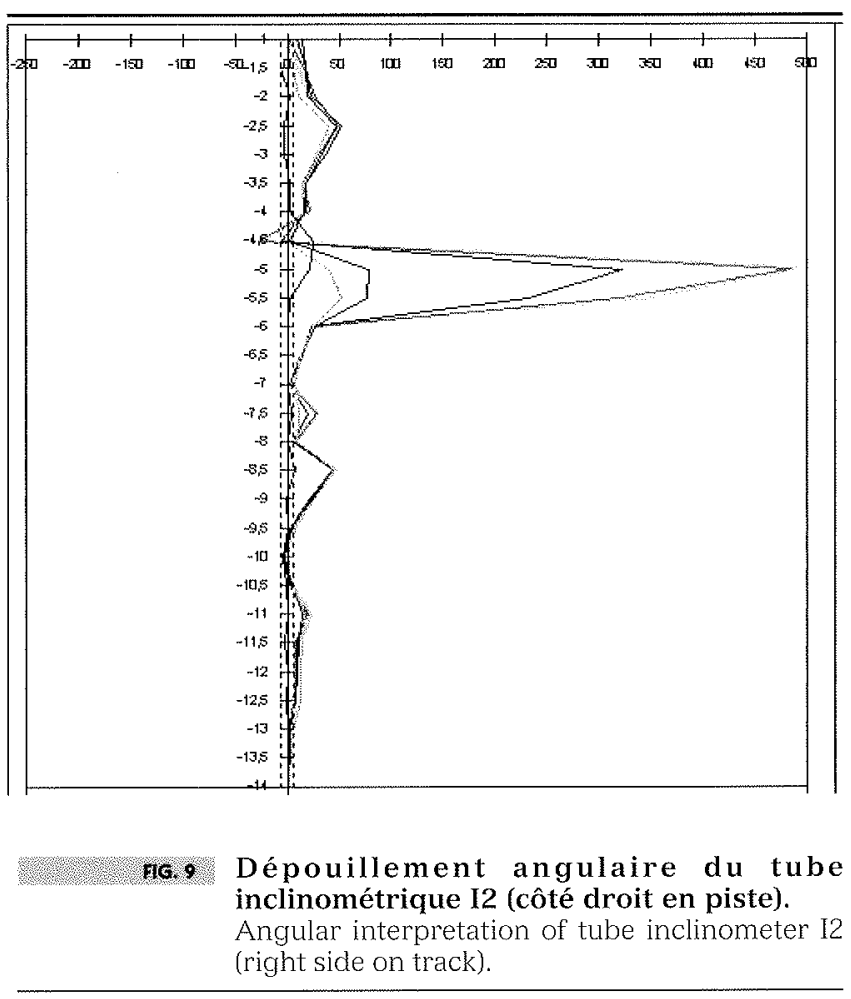

- contexte de glissement dans un versant instable avec existence d'un appareil de voie pris dans le glissement (nombreuses et coûteuses interventions pour reprendre les défauts) ;

- ligne à trafic important avec une vitesse des convois élevée $(150 \mathrm{~km} / \mathrm{h})$.

\section{8.}

Étude du confortement de l'ouvrage en terre

Une première phase est primordiale : avant de faire le choix du type de confortement à mettre en ouvre pour stabiliser les mouvements, il convient de réunir l'ensemble des données disponibles permettant d'éta- 


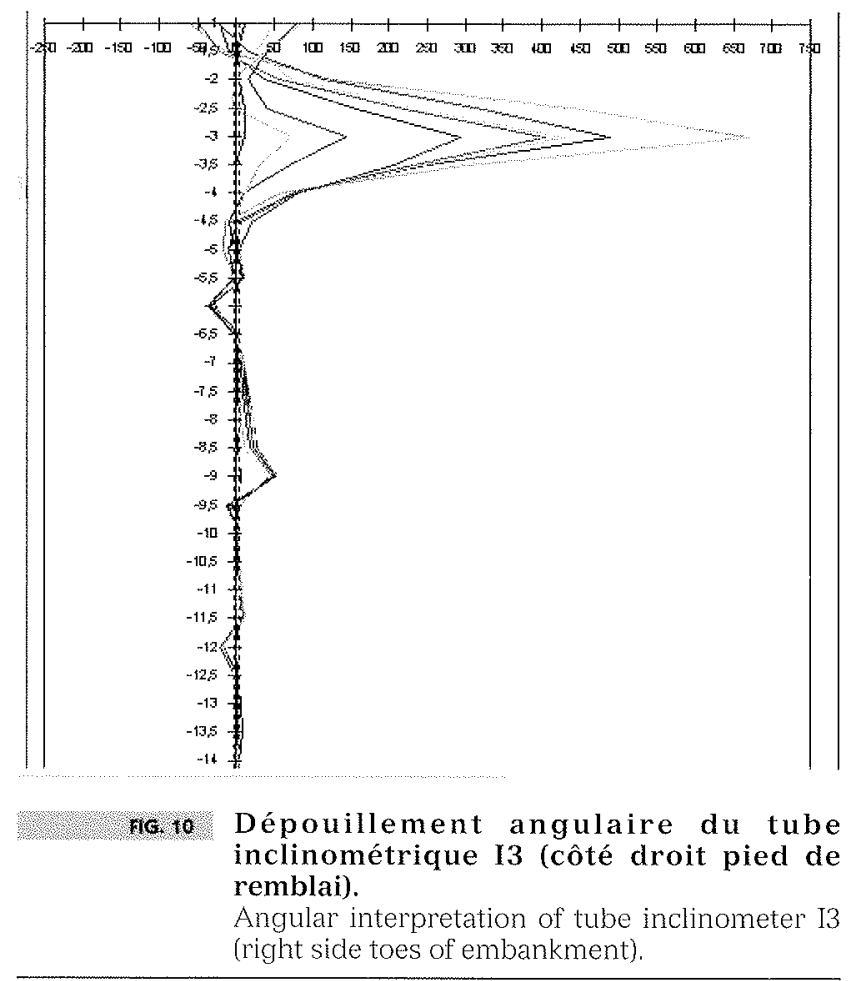

blir un modèle géotechnique représentatif du phénomène. Il faut disposer :

- des archives disponibles dans les différents services de maintenance, sur les désordres ayant affecté l'ouvrage par le passé, les investigations réalisées, les travaux entrepris (la fiche signalétique descriptive de l'ouvrage est une aide) ;

- des cartes géologiques et photos aériennes de la région;

- d'une vue en plan et des profils en travers représentatifs cle l'ouvrage ;

- des coupes de l'ensemble des sondages réalisés;

- des résultats des essais in situ et en laboratoire effectués sur les échantillons prélevés.

Cette analyse débouche sur la définition d'horizons géologiques homogènes reportés sur le ou les profils en travers les plus représentatifs, sur lesquels on note le niveau de la nappe à partir des résultats des mesures piézométriques et on place la surface de rupture affectant le versant à partir des résultats des mesures inclinométriques (Fig. 12).

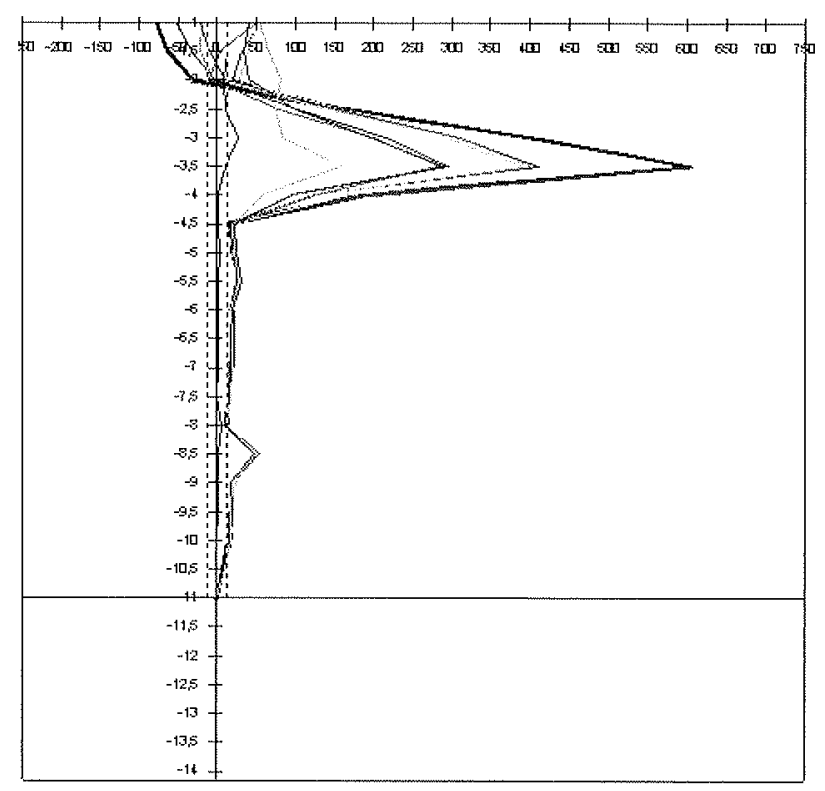

FG. 11 Dépouillement angulaire du tube inclinométrique 14 (côté droit dans le champ aval).

Angular interpretation of tube inclinometer I4 (right side in the field upstream)

\section{9.}

\section{Choix du type de confortement}

Un premier choix peut être ébauché avant l'étude, en fonction de la configuration du site et du contexte géologique et géotechnique mais il n'est détaillé qu'au cours de l'étude de phase projet (PRO) et doit être justifié. Dans certains cas plus complexes, en particulier lorsqu'une phase avant-projet (AVP) est prévue dans le déroulement du projet, deux solutions alternatives peuvent être étudiées. Il convient dans un premier temps d'écarter les solutions qui ne peuvent être envisagées dans le cas présent:

- la solution confortative par terrassement, la plus économique, est à examiner préférentiellement : cette solution (butée de pied avec bêche) n'est pas efficace dans le contexte de versant des Bas, sauf à envisager une bêche de plus de $4 \mathrm{~m}$ de profondeur en pied d'ouvrage, ce qui entraînerait d'importants risques de mouvement de la voie pendant les terrassements (même si l'on travaillait par plots), pouvant aller jusqu'à la rupture du

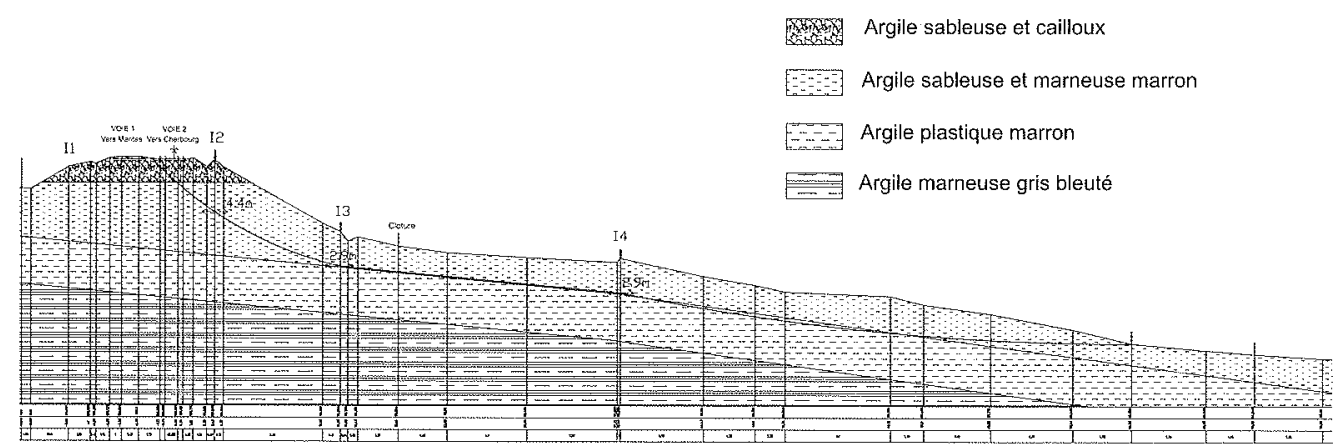

16. 12 Profil en travers géotechnique de synthèse.

Synthetic geotechnical profile. 
remblai, interrompant ainsi les circulations ferroviaires ;

- la solution drainage superficiel n'apporterait rien car un fossé béton en bon état et efficace est déjà présent en amont de la plate-forme ferroviaire ;

- des travaux de drainage profond par tranchée drainante amont seraient d'une efficacité médiocre compte tenu de la position de la nappe juste au-dessus de la surface de rupture; par ailleurs, cet aménagement entraînerait des contraintes d'entretien non négligeables et a une efficacité plus faible avec le temps;

- le clouage par pieux forés est une solution bien adaptée mais son coût est plus important que celui de la solution qui sera retenue (condition d'accès plus difficile pour une foreuse, nécessité de faire une banquette de travail, coût du béton de scellement).

Il a été finalement proposé un confortement par pieux verticaux battus; ce mode de confortement est bien adapté à un ouvrage sur un versant où le glissement affecte le sol support. Nous avons par ailleurs un bon retour d'expérience des travaux réalisés avec cette méthode dans les trente dernières années sur des sites contigus à l'ouvrage étudié.

Les terrains traversés sont consistants (modules pressiométriques en profondeur de l'ordre de $20 \mathrm{MPa}$ ) mais permettent néanmoins le battage de profilés. Cette technique est également bien adaptée car elle génère peu de contraintes vis-à-vis des circulations ferroviaires :

- faible impact sur la circulation des trains (toutefois, un ralentissement des convois à $80 \mathrm{~km} / \mathrm{h}$ au droit des travaux peut s'avérer nécessaire) ;

- accès par le pied de remblai hors contraintes ferroviaires ;

- pas de coupure du courant dans la caténaire.

\section{2.}

\section{Dimensionnement du confortement}

Le confortement de type clouage par pieux verticaux utilise la méthode des modules de réaction. Cette méthode se base sur l'hypothèse que la partie du massif en mouvement engendre par les efforts appliqués sur les clous une déformation de ceux-ci. Les efforts résultants (travail essentiellement en flexion) transmis par le pieu aux terrains sous la surface de rupture s'opposent ainsi aux déplacements. Il convient de résoudre l'équilibre des pressions sur le pieu :

$$
E I \frac{d^{4} y}{d z^{4}}=K_{s}[y(z)-g(z)]
$$

où : E est le module d'Young de l'acier ; I le module d'inertie du pieu; $\mathrm{K}_{\mathrm{s}}$ le module de réaction du sol; $\mathrm{y}(\mathrm{z})$ et $g(z)$ respectivement le déplacement horizontal du pieu et du sol en fonction de la profondeur (Fig. 13).

Quand le sol se déplace, il exerce une pression sur le pieu. Plus le déplacement est important plus l'effort sur le pieu est élevé et plus le pieu se déforme également. De par son inertie, il va se développer dans le pieu, au niveau de la surface de rupture, un effort résistant $\mathrm{T}$, qui est fonction du déplacement relatif sol-pieu $y(z)-g(z)$, et qui va s'opposer aux efforts moteurs.

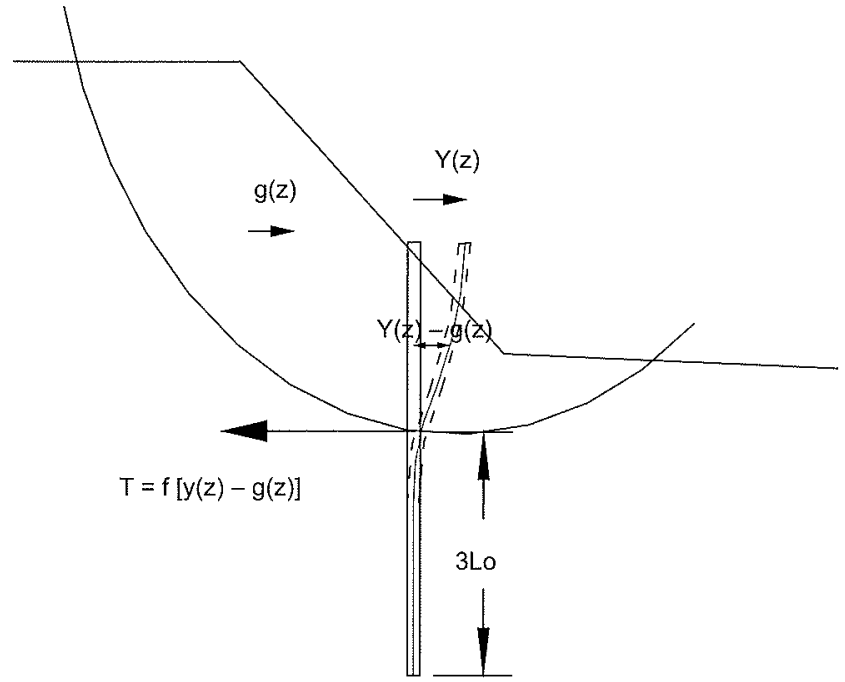

F6. 13 Dessin explicatif de la méthode des modules de réaction.

Explicative drawing of the method of reaction modulus.

La méthodologie de calcul utilisée consiste à travailler à rebours, sachant que l'on connait de façon assez précise la surface de rupture que l'on étudie. Ainsi, on cale les caractéristiques des différentes couches de sol pour obtenir un coefficient de sécurité $\mathrm{F}=\Sigma \mathrm{M}_{\text {res }} / \Sigma \mathrm{M}_{\text {mot }}$ proche de 1 (au-dessus de 1 car le glissement est actif mais l'ouvrage n'a pas atteint la rupture).

La cohésion des sols est prise égale à 0 dans les horizons traversés par la surface de rupture détectée et estimée avec une valeur raisonnable à l'extérieur de cette surface. L'angle de frottement pris en compte dans les calculs est estimé à partir d'une corrélation entre l'indice de plasticité du sol, Ip et l'angle de frottement effectif $\varphi^{\prime}$, tout en prenant compte de la nature des matériaux concernés, pour permettre un calage du coefficient de sécurité initial proche de 1,00. Les caractéristiques présentées dans le tableau I ont été calées pour l'exemple du remblai des Bas.

\begin{tabular}{|c|c|c|c|c|}
\hline \multirow{2}{*}{$\begin{array}{l}\text { TABLEAU } \\
\text { Conches }\end{array}$} & \multicolumn{4}{|c|}{$\begin{array}{l}\text { Caractéristiques des sols pour le remblai } \\
\text { des Bas. } \\
\text { Soils characteristics for Bas embankment. }\end{array}$} \\
\hline & & 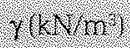 & $9 \cdot(\operatorname{cegr})$ & $C^{\prime}(\mathrm{RPa})$ \\
\hline \multicolumn{2}{|c|}{$\begin{array}{l}\text { Argile sableuse } \\
\text { + cailloux (remblai) }\end{array}$} & 20 & 20 & 0 \\
\hline \multicolumn{2}{|c|}{$\begin{array}{l}\text { Argile sableuse } \\
\text { et marneuse marron vert }\end{array}$} & 20 & 12 & 0 \\
\hline \multicolumn{2}{|c|}{ Argile plastique marron } & 20 & 22 & 5 \\
\hline \multicolumn{2}{|c|}{ Argile marneuse gris bleuté } & 20 & 22 & 10 \\
\hline
\end{tabular}

La caractéristique du sol permettant le dimensionnement du clouage est le module de réaction $\mathrm{K}_{\mathrm{s}}$ (qui est déterminé à partir d'essais pressiométriques-module pressiométrique $\mathrm{E}_{\mathrm{m}}$ et du diamètre du pieu) et la pression de fluage du sol qui est la pression maximale que l'on admet au contact sol pieu pour éviter tout fluage du sol aux abords du pieu. Les données d'entrées prises pour établir le dimensionnement du clouage sont présentées dans le tableau II. 
taBteau il Données d'entrées prises pour établir le dimensionnement du clouage. Input data for the nailing design.

\begin{tabular}{|c|c|c|c|c|c|c|c|c|}
\hline Srots & Proronaent (n) & I $k P a)$ & 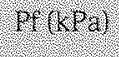 & $1(k) a)$ & a & $\mathrm{N},(\mathrm{N}) \mathrm{m}, \mathrm{r})$ & vitivis & 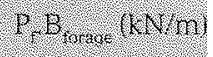 \\
\hline $\begin{array}{l}\text { Argile sableuse } \\
\text { et marneuse marron }\end{array}$ & 0 à $5,2 \mathrm{~m}$ & 1600 & 130 & 250 & 0,50 & 4867 & $26.6 \mathrm{E}-3$ & 110 \\
\hline Argile plastique & de $5,2 \mathrm{~m}$ à $9 \mathrm{~m}$ & 10000 & 400 & 900 & 0,67 & 24050 & $16.6 \mathrm{E}-3$ & 339 \\
\hline
\end{tabular}

L'objectif du confortement est d'améliorer la stabilité du glissement (surface de rupture initiale) par diminution des efforts moteurs du glissement de $20 \%$ soit une augmèntation du coefficient de sécurité F de $25 \%$ environ. Cette amélioration est liée à la mobilisation d'un effort de cisaillement dans le pieu au niveau de la surface de rupture (Figs. 14 et 15). Une vérification de la stabilité du glissement à l'aval du clouage est néces- saire pour s'assurer qu'aucun risque de décollement à l'aval du clouage ne peut se produire (Fig. 16); avec un coefficient de sécurité de 1,32 pour un glissement se développant sous le confortement, on peut dire que la stabilité est acquise. Dans le cas contraire, on aurait été obligé de vérifier le clouage en soutènement, ou d'ajouter un deuxième niveau de clouage.

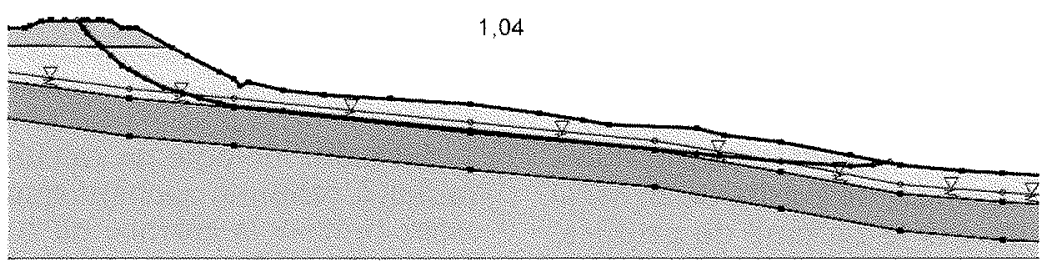

Méthade de calkul : Petturations

Système çe jondêration: Coefficients TAL $\mathrm{Fmin}=1.04$

16. 14. Calage des caractéristiques pour le facteur de sécurité $F$ proche de 1.

Characteristics calibration for the security factor close to 1.

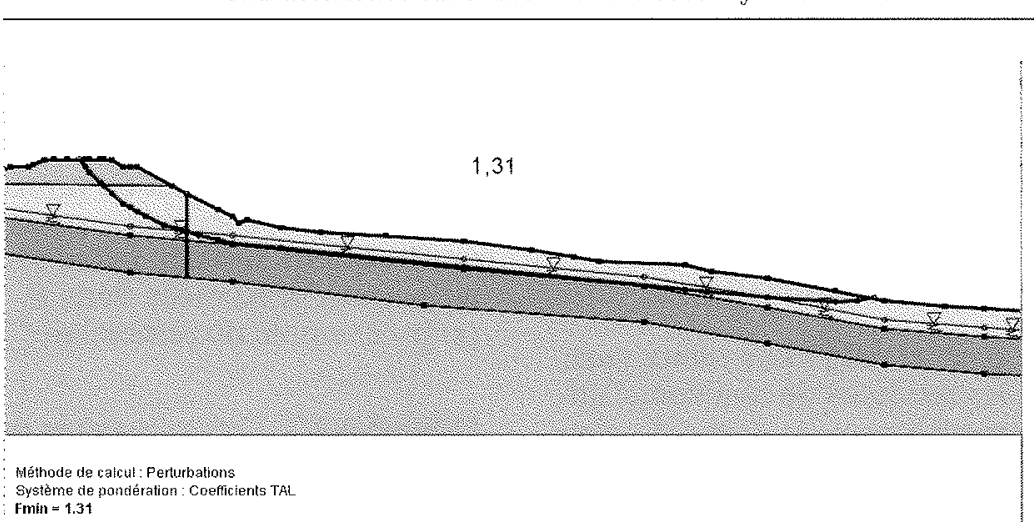

156. 15. Choix du maillage des pieux pour une amélioration de $\mathrm{F}$ de plus de $25 \%$. Selection of piles mesh for an improvement of $\mathrm{F}$ by $25 \%$. 
Le choix du confortement s'est porté sur des palplanches unitaires PAU2440 :

- épaisseur : e $=6 \mathrm{~mm}$;

- largeur : 0,848 m;

- inertie : $\mathrm{I}=9604.10^{-8} \mathrm{~m}^{4}$;

- moment statique $: \mathrm{I} / \mathrm{v}=651.10^{-6} \mathrm{~m}^{3}$;

- masse linéique $: m=58,3 \mathrm{~kg} / \mathrm{ml}$.

Lors de l'étude, un certain nombre de types de palplanches ont été testées de façon à optimiser le poids de l'acier tout en faisant travailler le profil à son maximum (en termes d'effort maximum et de moment maximum). La méthode consiste à l'aide du logiciel PILATE (LCPC) de laisser se déplacer le sol au-dessus de la surface de rupture de façon homogène pour atteindre le fluage (en général au niveau de cette surface) tout en ne dépassant pas une amplitude équivalente à deux ans de déplacement inclinométrique (durée maximum acceptable pour observer des mouvements résiduels après travaux).

Une palplanche reprend, d'après le calcul PILATE, $110 \mathrm{kN}$ ce qui permet de donner le maillage des profilés. On privilégiera une répartition sur plusieurs files pour s'assurer qu'aucune rétention d'eau ne se produit à l'arrière des palplanches et qu'un effet de groupe se développe au sein des pieux. On se reportera aux courbes de la figure 17. Le type d'acier (Nuance) est vérifié en calculant la contrainte maximum qui s'exerce sur la palplanche : $\sigma=$ moment maximum/inertie du profilé/v (rayon de giration du profilé).

Le calcul permet de définir le maillage des pieux du confortement soit pour le remblai des Bas, un pieu tous les 2,28 m sur 3 lignes espacées de 1,50 m (voir profil confortatif en figure 18).

\section{Travaux}

Les travaux de confortement ont été réalisés en mai 2005 ; ils ont duré environ deux mois. Ils ont nécessité :

- la création d'une piste d'accès ;

- la mise en place d'une grue ;

- l'utilisation d'un vibrofonceur pour la mise en fiche des palplanches et d'un marteau trépideur pour le surbattage ;

- la construction d'un guide pour obtenir une bonne verticalité des profilés.

Pendant les travaux, des mesures de vibrations ont été effectuées par un laboratoire indépendant (présence d'un aqueduc à proximité du chantier) et le suivi du nivellement des voies a été renforcé. Aucune anomalie particulière n'a été rencontrée au cours de ces suivis. Quelques problèmes de refus prématurés se sont produits liés à la consistance assez raide des matériaux du substratum et à la finesse des palplanches choisies pour le confortement (Fig. 19).

\section{9}

\section{Suivis après travaux}

Dans tout confortement et en particulier pour les confortements par pieux, où des mouvements résiduels sont observés après travaux en liaison avec le principe de confortement (module de réaction), il est très important de continuer le suivi des mouvements aussi bien sur la voie qu'au niveau de l'instrumentation inclinométrique.



Courbes de l'effort tranchant et du moment en fonction de la profondeur Effort tranchant ( $\mathrm{kN}$ ) / Moment ( $\mathrm{kN} . \mathrm{m}$ )
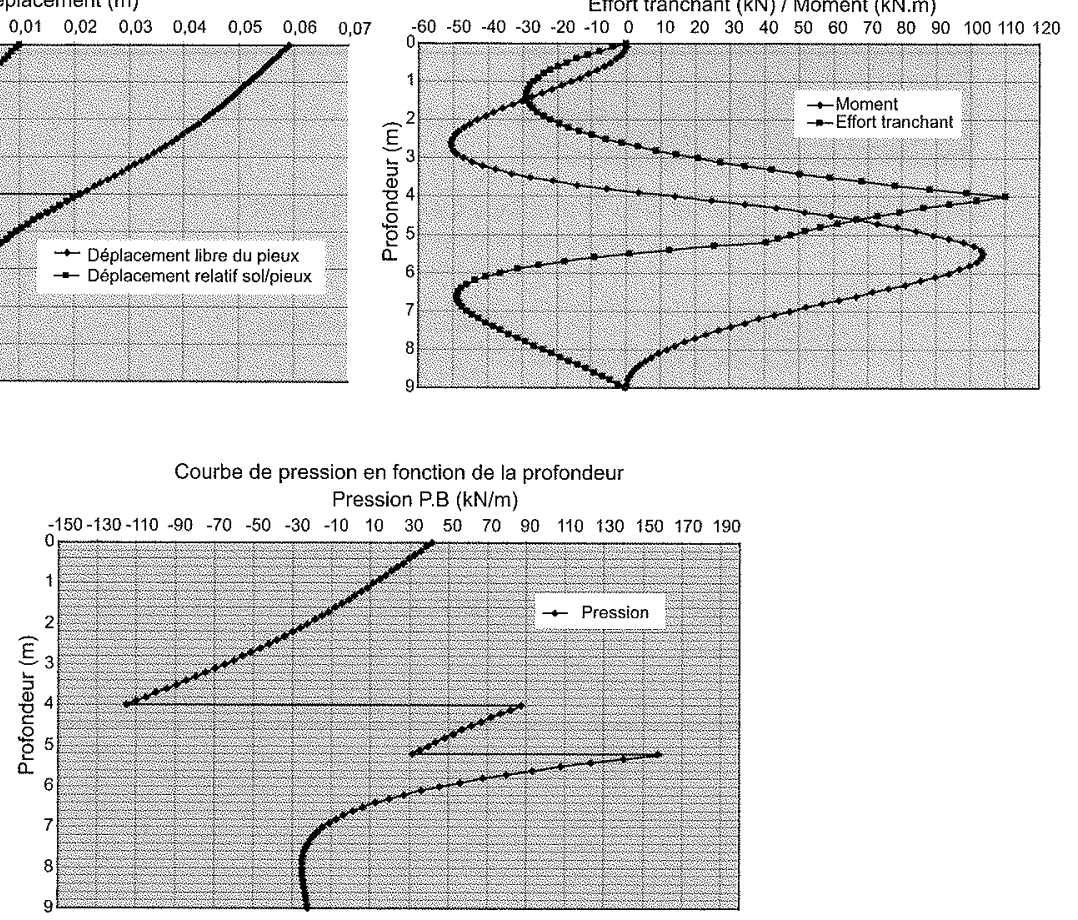

ric. 17 Résultats du calcul PILATE.

Results from calculation using PILATE. 
RO - Ligne $n^{\circ} 366$ : remblai des Bas

Profil de confortement type du km 203,008 au km 203,045

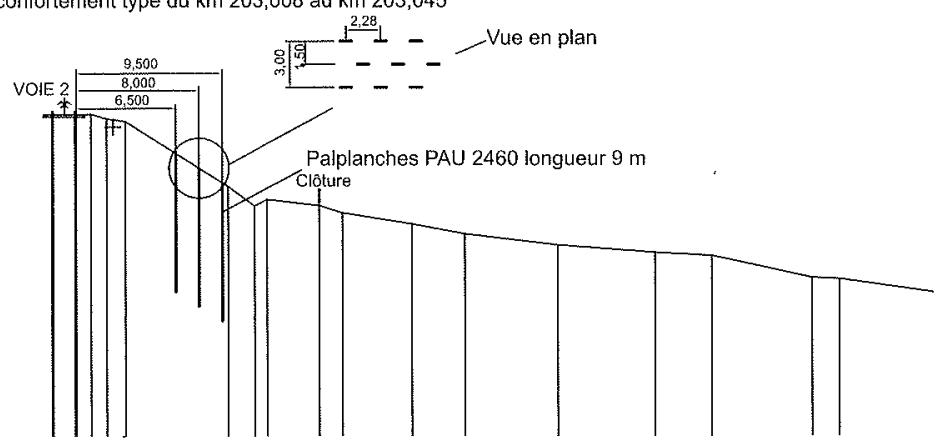

.16. 18. Profil en travers confortatif. Reinforcement profile.

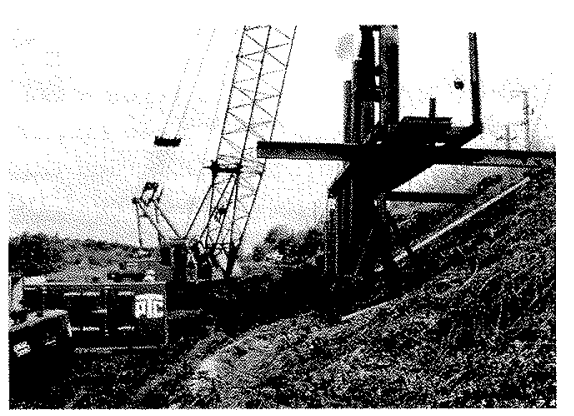

Vue générale du chantier avec la grue mettant en cuvre les palplanches.

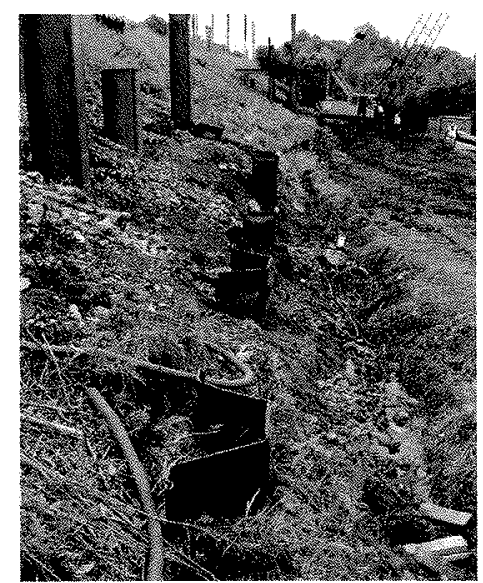

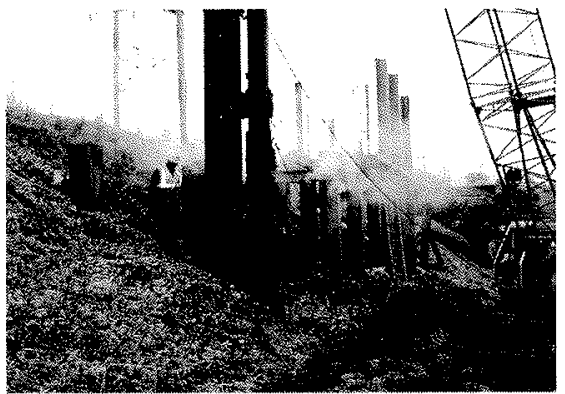

Battage en cours.



Travaux de battage terminés.

1. If 19 Photos des travaux de confortement. Pictures of the reinforcement work.
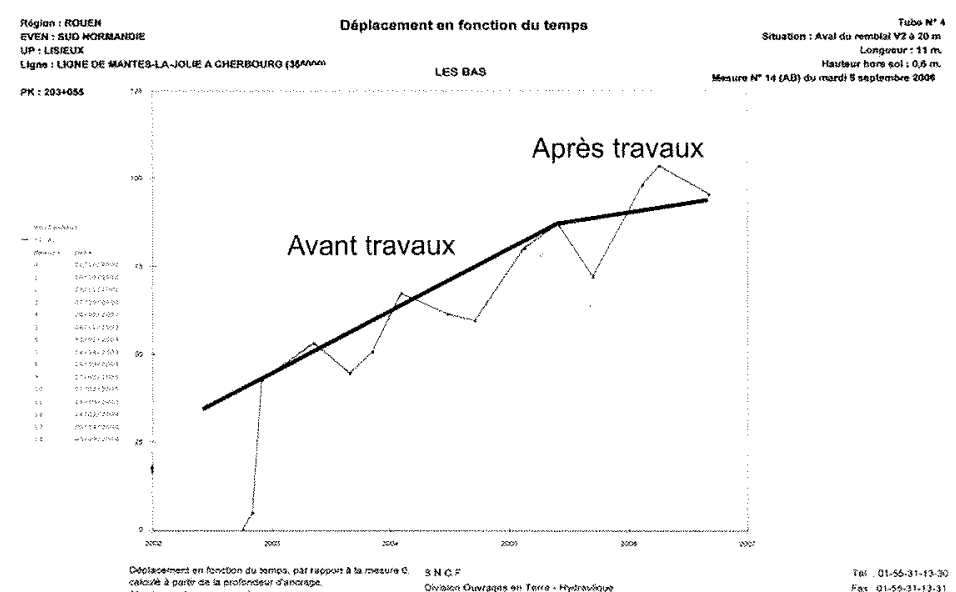

3सas

ศ6. 20. Suivi après travaux de confortement. Monitoring after reinforcement work. 
Depuis la mise en œuvre du confortement, aucune reprise de nivellement n'a été constatée sur la voie 2 qui était très sollicitée par le glissement avant le traitement et les mouvements inclinométriques ont été divisés par 5 à 10.

\section{3}

\section{Conclusion}

Les travaux réalisés sur le site du remblai des Bas ont montré une bonne stabilisation des mouvements affectant le site. La solution confortative était bien adaptée, d'une part, à la configuration du site et, d'autre part, à la géométrie du glissement et son dimensionnement est justifié par cette stabilisation. Actuellement, la SNCF est à la recherche d'une optimisation de la solution confortative, l'augmentation du coefficient de sécurité initial de $25 \%$ étant supérieure aux augmentations préconisées par le LCPC dans son guide de stabilisation des glissements de terrain. Le retour d'expérience actuel sur le réseau ferré national montre qu'avec ce dimensionnement, plus de $90 \%$ des sites confortés voient leur mouvement se stabiliser conformément à ce que les calculs prédisent.

\section{Bibliographie}

Collectif - Renforcement des sols par clouage. Bulletin de liaison des Ponts et Chaussées, 1988.

Durville. J.-L., Seve. G. - Stabilité des pentes. Glissements en terrain meuble. Techniques de l'ingénieur, C254, 1996
Frank. R. - Fondations profondes. Techniques de l'ingénieur, C248, 1995.

Frank. R. - Comportement d'un pieu isolé sous effort de flexion. Techniques de l'ingénieur, C249.
Pouget. P., Seve G. - Stabilisation des glissements de terrains. Guide technique LCPC, 1998.

Schlosser. F., Unterreiner. P. -- Renforcement des sols par inclusions. Techniques de l'ingénieur, C245, 1994. 